\title{
Structural Characterization of an Enantiopure Hydroxo-bridged Binuclear Iron(III) Complex with Empty One-dimensional Helical Channels
}

Md. Akhtarul Alam†, Munirathinum Nethajił*, and Manabendra Ray†*

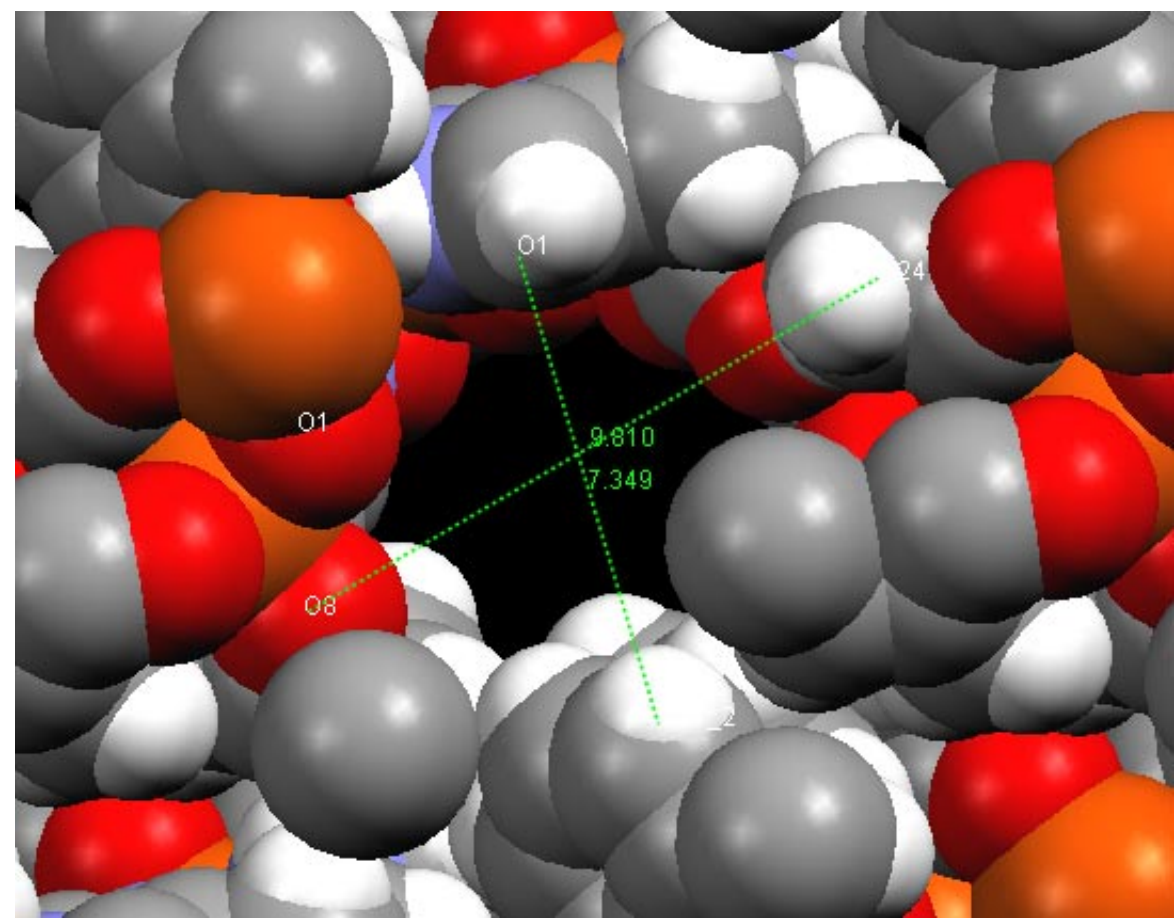

Figure S1. The cavity diameter in 1 was calculated by atom-atom distances between O8-C24 and $\mathrm{O} 1-\mathrm{C} 11$.
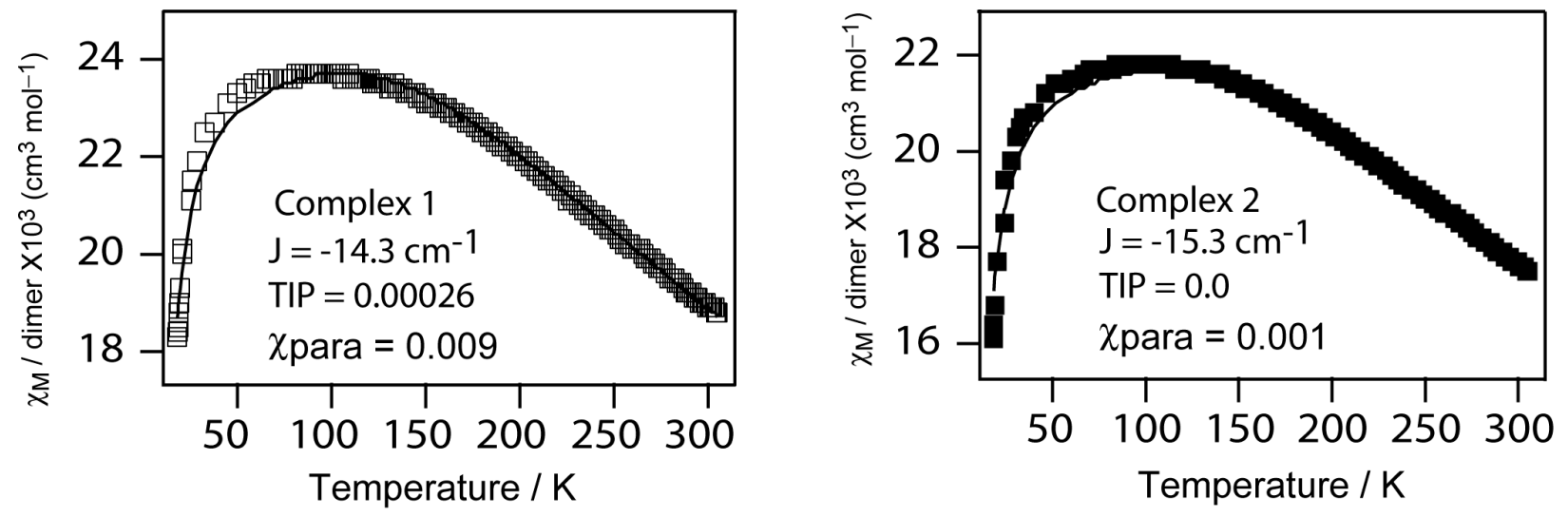

Figure S2. The magnetic susceptibility plots with parameters used in the fit. The fitting equation was taken from J. Chem. Soc., Dalton Trans., 2001, 2616 where g was kept fixed at 2.00 and $\Theta$ was taken as zero. 
S2

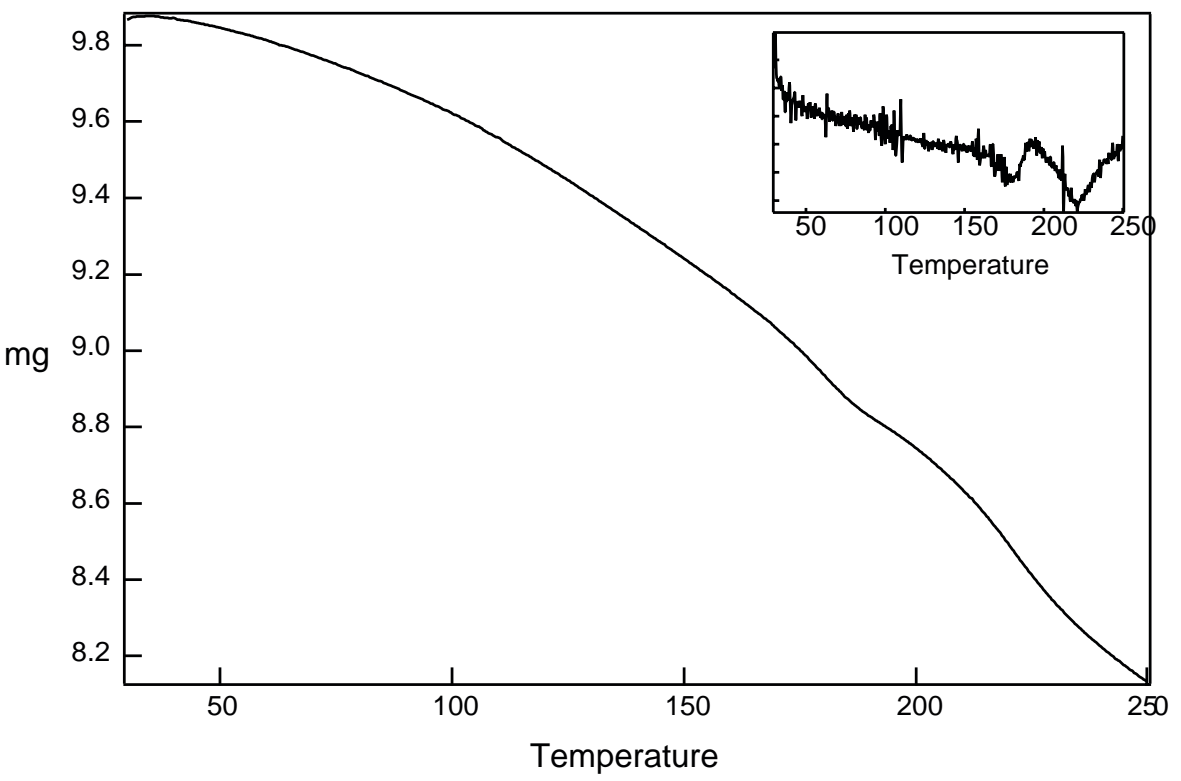

Figure S3. TGA plot of iodine doped 2 with derivative (DTA) plot of TGA as inset. 


\section{Rehydration of the dried crystals}
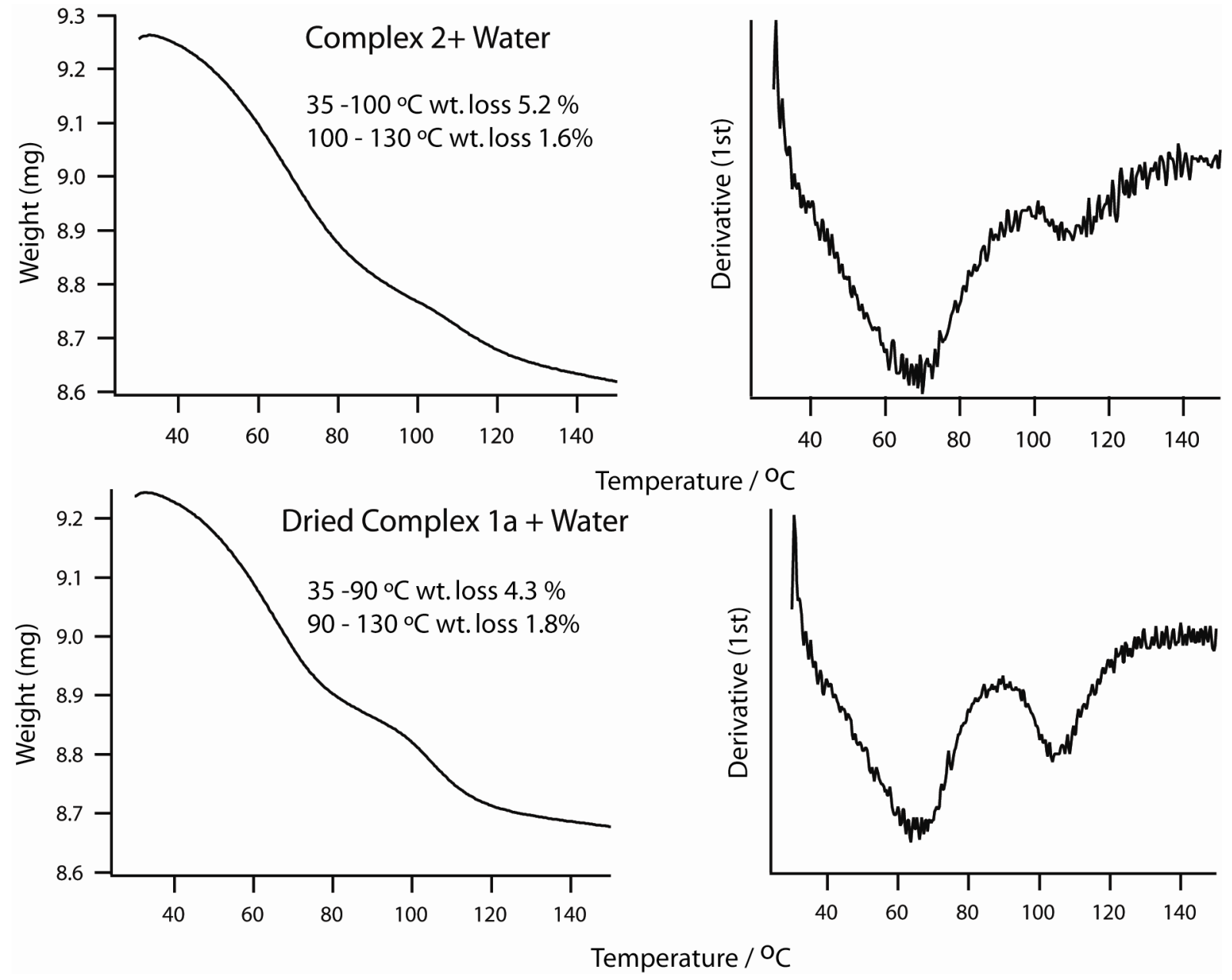

Figure S4. TGA (left) and DTA (right) plot of 2 and $1 \mathbf{a}$ (dried at $90^{\circ} \mathrm{C} 2 \mathrm{~h}$ ) after exposure to water vapor for three days. Longer exposure to water does not have any effect on weight loss. 
Figure $\mathrm{S} 5$ showing the $3 \mathrm{H}_{2} \mathrm{O}$ waters (disordered in 1) and $1 \mathrm{H}_{2} \mathrm{O}$ in the channels of $\mathbf{1}$ and $\mathbf{1 a}$ respectively. Table $\mathrm{S} 1$ have the bond parameters and occupancy used while solving the structure of 1 .

A

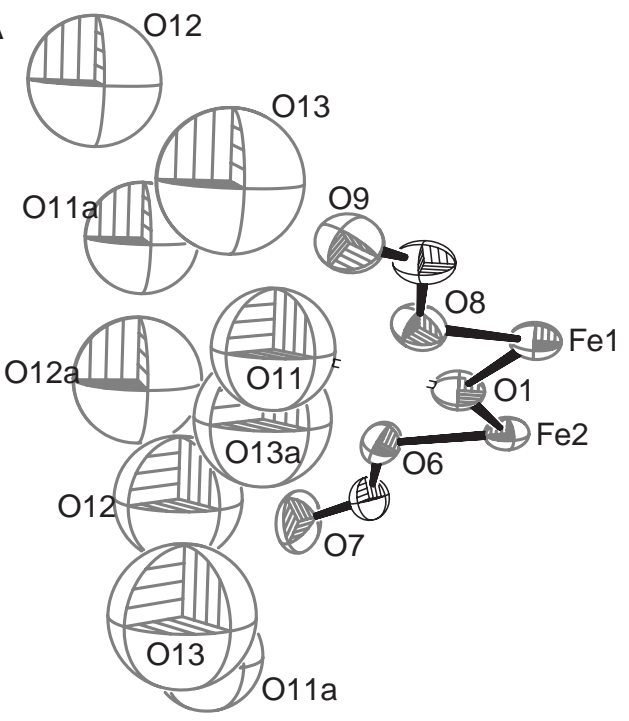

B 09

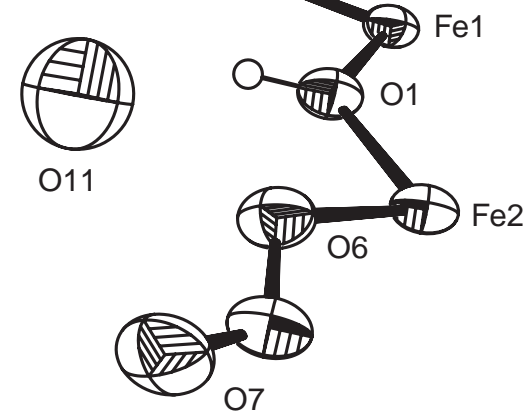

Figure S5. Water molecules in the channel of $\mathbf{1}(\mathrm{A})$ and $\mathbf{1 a}(\mathrm{B})$.

Table-S1

\begin{tabular}{lcll}
\hline Atom & Occupancy & & Distances $(\AA)$ \\
\hline In 1 & & & \\
O11 & 0.6 & O11-O11a & $2.6002(0.0302)$ \\
O11a & 0.4 & O11-O12a & $2.9682(0.0275)$ \\
O12 & 0.5 & O11-O13 & $2.4368(0.0311)$ \\
O12a & 0.5 & O11a-O13a & $3.1709(0.0366)$ \\
O13 & 0.6 & O12a-O13a & $2.7710(0.0400)$ \\
O13a & 0.4 & O1-O13a & $2.7850(0.0479)$ \\
& & O13-O13a & $3.3874(0.0419)$ \\
& & O11-O12 & $3.1002(0.0301)$
\end{tabular}

In $1 \mathbf{a}$

O11 $\quad 1.0 \quad 01-011 \quad 2.790$


A

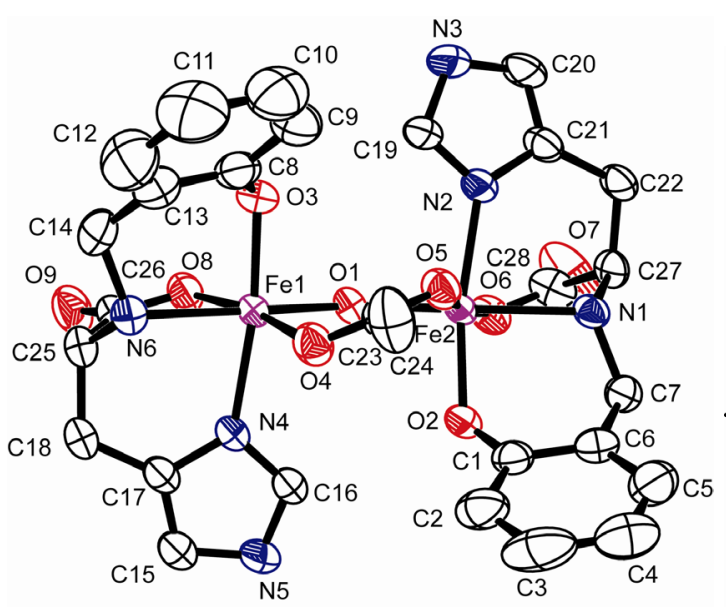

B

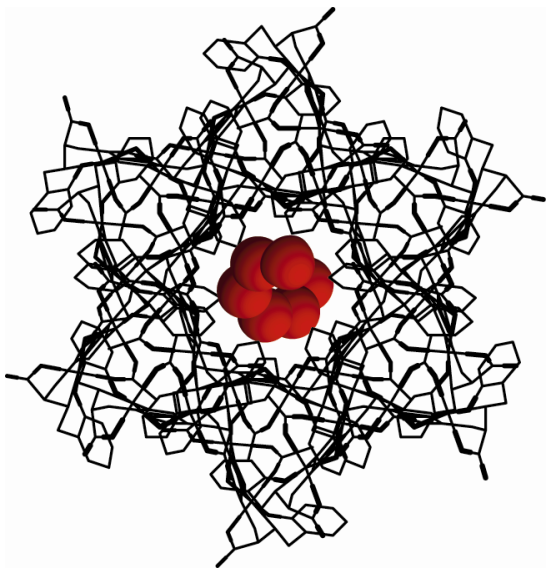

Figure S6. (a) ORTEP diagram of the complex 1a (b) left handed helix formed by $\mathbf{1 a}$.

A

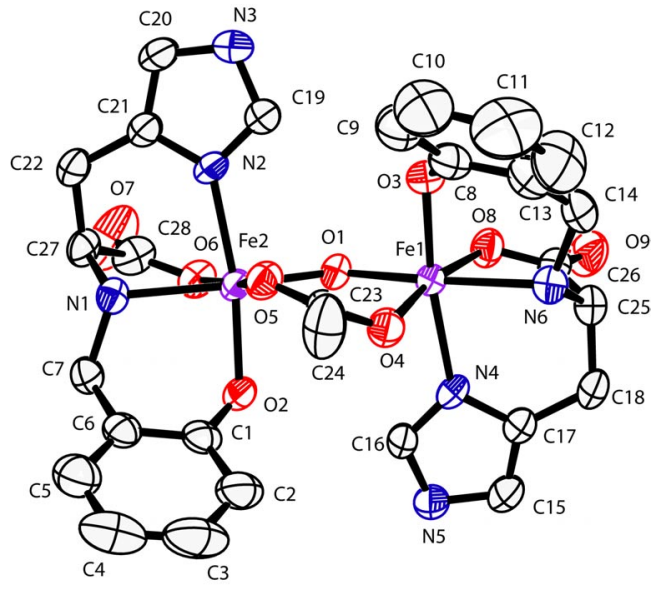

B

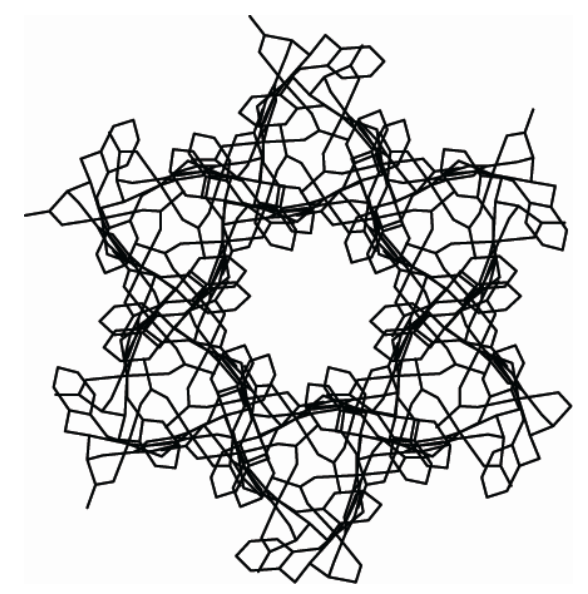

Figure S7. (a) ORTEP diagram of the complex 2 (b) Empty channel of 2. 
A

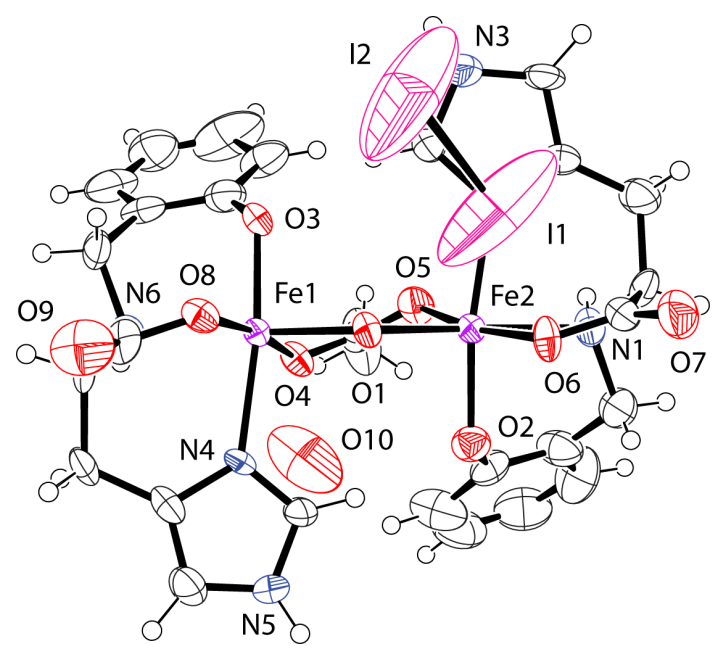

B

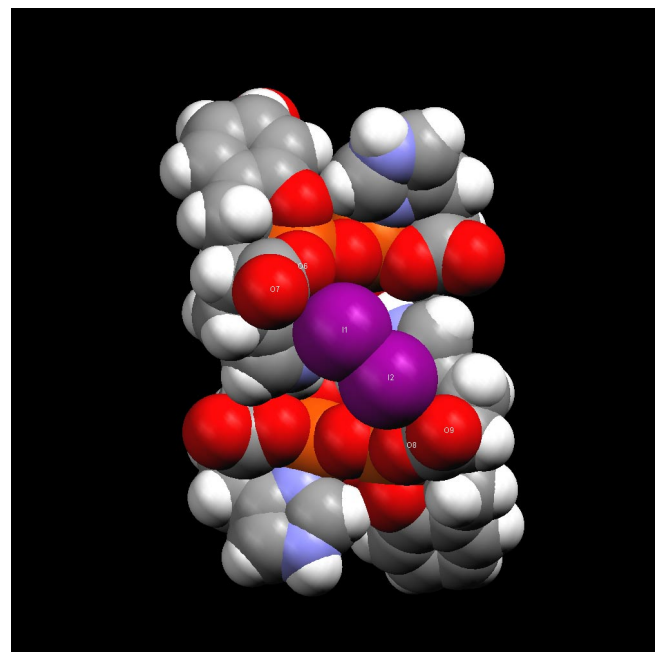

Figure S8. (a) ORTEP diagram of the complex 3 (b) Iodine molecules between two dimers in 3. 